\author{
RESEARCH ARTICLE \\ 10.1029/2019JC015717 \\ Key Points: \\ - Wind properties are estimated based \\ on a $f^{-4}$ spectral dependence in the \\ equilibrium range \\ - Wind speed and direction can \\ be estimated based on wave \\ measurements alone with reasonable \\ accuracy \\ - Estimates of wind properties can \\ be improved by reducing the \\ dimensions of the wave buoy
}

Supporting Information:

- Supporting Information S1

Correspondence to:

J. J. Voermans,

jvoermans@unimelb.edu.au

Citation:

Voermans J. J., Smit, P. B., Janssen, T., \& Babanin, A. V. (2020). Estimating wind speed and direction using wave spectra. Journal of Geophysical Research: Oceans, 125, e2019JC015717. https://doi.org/10.1029/2019JC015717

Received 30 SEP 2019

Accepted 30 JAN 2020

Accepted article online 5 FEB 2020

(C)2020. American Geophysical Union. All Rights Reserved.

\section{Estimating Wind Speed and Direction Using Wave Spectra}

\author{
J. J. Voermans ${ }^{1}(\mathbb{D})$, P. B. Smit ${ }^{2}$ iD, T. T. Janssen ${ }^{2}$ (D) and A. V. Babanin ${ }^{1,3}$ (D) \\ ${ }^{1}$ Department of Infrastructure Engineering, University of Melbourne, Melbourne, Victoria, Australia, ${ }^{2}$ Sofar Ocean \\ Technologies, San Francisco, CA, USA, ${ }^{3}$ Laboratory for Regional Oceanography and Numerical Modeling, Qingdao \\ National Laboratory for Marine Science and Technology, Qingdao, China
}

\begin{abstract}
Compact wave buoys are increasingly used to provide monitoring of coastal and oceanic conditions by measuring surface waves in real time. Due to their relatively compact size, they are generally not suited to measure wind directly. However, since the wave field is intrinsically coupled to the wind field, wave measurements can serve as a proxy observation of ocean surface winds. In this study, we use spectral wave observations to estimate wind speed and direction based on the assumption of a universal $f^{-4}$ shape of the wave energy spectrum in the equilibrium range and the presence of a logarithmic wind speed profile above the ocean surface. The wind speed and direction were estimated between 2014 and 2017 at more than a 100 coastal sites with colocated wave and wind observations. Estimates of wind speed and direction based on wave measurements have a root-mean-square error of $2 \mathrm{~m} / \mathrm{s}$ for wind speeds between 3 and $12 \mathrm{~m} / \mathrm{s}$ (and a relative error of $17 \%$ for wind speeds between 10 and $20 \mathrm{~m} / \mathrm{s}$ ) and up to $20^{\circ}$ for wind speeds between 10 and $20 \mathrm{~m} / \mathrm{s}$. The accuracy of proxy measurements of wind depends on fetch, wave steepness, wave age, directional alignment between wind and dominant waves, and temporal variability of the wind. Further, we show that estimates of wind speed and direction improve considerably as the size of the buoy is reduced.
\end{abstract}

Plain Language Summary Coastal marine weather conditions are continuously monitored by meteorological buoys and satellites to inform the public, authorities, and emergency services. However, observations of wind and waves from meteorological buoys and satellites are strongly restricted by either their spatial or temporal resolution. Wave buoys, which measure waves only and not wind, can be more compact, which makes them easier to deploy, relatively low cost, and therefore much better suited to deploy in larger numbers. Here, we explore whether we can exploit the coupling of short surface waves to the near-surface wind to quantitatively estimate surface wind properties based on wave measurements. We use an extensive data set of collocated wind and wave measurements to show that measurements of short wind waves can indeed provide a useful estimate of wind speed and direction. We also show that the accuracy of the estimates are considerably improved when the size of the buoy is reduced. The fidelity of surface wind estimates based on wave measurements is comparable to estimates based on remote sensing satellite data. This suggests that networks of wave buoys can successfully provide real-time and long-dwell estimates of surface wind speed and direction and thus successfully augment available meteorological buoy networks and satellite data.

\section{Introduction}

Collocated observations of surface waves and winds are important for understanding and modeling of many coastal processes and predicting coastal hazards (e.g., Bidlot et al., 2002). Such collocated observations are either provided by networks of in situ meteorological buoys, sometimes augmented by satellite remote sensing data. However, meteorological buoy networks are exceedingly sparse, and satellite observations are limited in their temporal resolutions (typically once or twice a day) and often not available near coastal boundaries due to backscatter contamination from land (e.g., Pickett et al., 2003). As a result, collocated wind and wave observations are often not available, and nearly everywhere quite sparse.

The most reliable and accurate measurements for near-surface ocean weather (waves and wind) are provided by in situ meteorological buoys. To provide a suitable platform for an anemometer mast, these buoys are relatively large and expensive. Due to their size, their deployment and maintenance operations require vessels with sufficient lifting capacity and robust mooring systems. Moreover, the larger platform size will 
negatively affect the buoy response to shorter waves. As a result of the expense and operational complexity of these systems, these buoy networks are generally sparely available along coastlines of developed countries and practically nonexistent in the rest of the world.

In contrast, wave buoys are much simpler devices, and without the elevated anemometer, they can be much more compact and of considerably lower cost (Raghukumar et al., 2019). These smaller systems can use simple and more lightweight mooring systems, and the smallest versions can be readily deployed by hand from small vessels or even airplanes. Moreover, due to their smaller size, these systems generally can track much shorter waves due to reduced inertia and superior surface-following properties. However, without an elevated anemometer, these devices cannot directly measure the wind properties. Since surface waves are driven by the surface winds imparting momentum and energy on the upper ocean, the wave field may provide a suitable proxy for the surface winds, possibly of similar accuracy as satellite remote sensing, or better (Thomson et al., 2013). If that is the case, networks of wave buoys could augment networks of meteorological buoys and satellite remote sensing.

To understand the nature of the proxy provided by the wave field, consider the following. The majority of momentum transfer from the wind to the surface waves occurs at the high-frequency end of the wave energy spectrum $E(f)$ (where $f$ is the frequency), also referred to as the spectral tail. As a result, the momentum transfer (or wind stress) directly affects the form of the spectral tail (Makin et al., 1995). Specifically, for $1.3 f_{p} \lesssim f \lesssim 3 f_{p}$ (with $f_{p}$ the peak frequency) spectral energy has been found to be of the form $E(f) \propto$ $u_{*} f^{-4}$ (Toba, 1973), relating spectral energy levels linearly to the friction velocity $u_{*}$ and thus to the wind stress ( $\tau=\rho u_{*}^{2}$, with $\rho$ density). This region of the spectral tail is often referred to as the equilibrium range of the wave spectrum, because when equilibrium is achieved, spectral levels in the equilibrium range are entirely determined by the balance between generation (wind), redistribution (nonlinear interactions), and dissipation (breaking).

In general then, the spectral tail evolves under the effects of wave generation by wind $S_{i n}$, dissipation by wave breaking $S_{\text {diss }}$ and nonlinear wave-wave interaction $S_{n l}$, which may be approximated through a spectral energy balance of the form (e.g., Holthuijsen, 2010):

$$
\frac{\mathrm{d} E}{\mathrm{~d} t}=S_{i n}+S_{n l}+S_{d i s s}
$$

where $E(f)$ is the spectral energy density in the frequency domain $f$ and all terms in equation (1) depend on $E(f)$.

The tail of the spectrum responds relatively rapidly to changing wind conditions and in the open ocean relaxes to an equilibrium solution for which $\mathrm{d} E / \mathrm{d} t \approx 0$. If, following Phillips (1985), the wind forcing is assumed homogeneous and stationary the spectrum takes the equilibrium shape:

$$
E(f)=E_{0} f^{-4} \text { with } E_{0}=\frac{4 \beta I u_{*} g}{(2 \pi)^{3}} f>1.3 f_{p}
$$

where $\beta$ is an empirical constant. Further, $I(p)$ was introduced to allow for enhanced tail energy levels when directional spreading of the waves is low and ranges from 1.9 for wide directional spreads to 3.1 for narrow waves (Juszko et al., 1995). A constant value if $I(p)=2.5$ (corresponding to a spread of order $30^{\circ}$ ) was found to give a reasonable approximation for a wide variety of observations (Thomson et al., 2013) and in what follows we will set $I(p)=2.5$. To relate $u_{*}$ values to surface wind speeds, we assume a logarithmic wind speed profile $U(z)$ so that

$$
U(z)=\frac{u_{*}}{\kappa} \ln \left(\frac{z}{z_{0}}\right),
$$

where $\kappa=0.41$ is the Von Karman constant and $z_{0}$ is the ocean surface roughness length, which is typically parametrized as (Edson et al., 2013)

$$
z_{0}=0.11 \frac{v}{u_{*}}+\alpha \frac{u_{*}^{2}}{g} .
$$

Here, $v$ is the kinematic viscosity of air and $\alpha$ is the Charnock parameter (Charnock, 1955; Edson et al., 2013). The first term in equation (4) represents the limit of a smooth surface defined by the thickness of the 


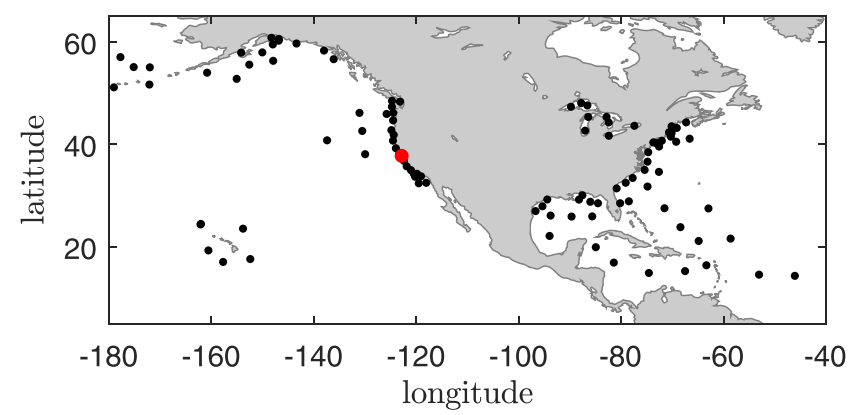

Figure 1. Distribution of the 102 NDBC buoys considered in this study. The Spotter buoy is located next to NDBC 46026, marked in red.

viscous sublayer, while the second term is due to form drag. For wind speeds larger than about $7 \mathrm{~m} / \mathrm{s}$, the surface is generally fully rough, in which case roughness length is determined by form drag.

Equations (2)-(4) provide a direct relation between energy levels in the equilibrium range of the wave spec$\operatorname{trum}\left(E_{0}\right)$ and the near-surface wind speed profile $(U(z))$, provided that values for model parameters $\beta, I(p)$ and $\alpha$ are known. Common values are $\beta(\approx 0.012$, Juszko et al., 1995), $\alpha(\approx 0.012$, Charnock, 1955), and $I(p)(\approx 2.5$, Thomson et al., 2013), but it is expected that optimal values for this inversion depends on the observation platform used and the associated response to the surface wave field.

To be clear, the dynamics of the wave spectral tail are not yet fully understood, and the relative importance of individual source terms is still debated (e.g., $f^{-4}$ can be explained by nonlinear interactions alone, see, e.g., Kitaigorodskii, 1983). However, the $f^{-4}$ shape in the equilibrium range and its linear dependence on $u_{*}$ are well supported by observations (e.g., Lenain \& Melville, 2017), and fitting $E_{0} f^{-4}$ to observed spectra and using (2) to estimate $u_{*}$ has been shown to approximate wind stress observations well (Thomson et al., 2013). Note that for frequencies beyond the equilibrium range $\left(f>3 f_{p}\right)$, dissipation is likely to saturate the spectrum and a transition to $f^{-5}$ is often observed (Babanin, 2010; Forristall, 1981; Hansen et al., 1990).

The objective of the present work is not to discuss the intricacies of the spectral tail but rather focus on the estimation of ocean surface winds based on observed energy levels in the equilibrium range of wave spectra measured by wave-following surface buoys. To that end, we consider an extensive set of collocated wind and wave observations from the National Data Buoy Center (NDBC); see section 2.1 In our method, we use a momentum balance to estimate $\beta$ and $\alpha$ directly from observations of wind speed $U$ (see section 2.2) and use these estimates to derive wind speeds and directions from observed energy levels in the equilibrium range (see section 2.3). Our principal results are presented in section 3. In section 4 we discuss the limits of our model, sensitivities, observed variations between sites, and form factor of the observing platform. We summarize our findings in section 5 .

\section{Methods}

\subsection{Observational Data}

The wave and wind observations used in this study are collected by the NDBC. The data set used here covers the time period 2014-2017 and includes 102 buoys in coastal and oceanic regions, as well as inland waters (Figure 1) where collocated wind and wave data were available. Wind speed and direction is typically measured at 4-5 $\mathrm{m}$ above mean sea level and has an accuracy of $\pm 1 \mathrm{~m} / \mathrm{s}$ and $\pm 10^{\circ}$, respectively. For this study, measured wind speed is extrapolated to the standard height of $10 \mathrm{~m}$ using the power law $U_{10} / U_{a}=\left(10 / z_{a}\right)^{0.11}$ (Hsu et al., 1994), where $U_{a}$ is the wind speed measured by the anemometer at height $z_{a}$. Wave energy is available in the range $0.02-0.485 \mathrm{~Hz}$, and the accuracy of the measured significant wave height is approximately $\pm 0.2 \mathrm{~m}$. The preprocessed data set comprises nearly 2.9 million sets of measurements.

To determine the effect of the buoy form factor on the ability to measure the equilibrium range and thus surface wind dynamics, we also considered a Spotter wave buoy (Raghukumar et al., 2019) that was deployed near NDBC Buoy 46026 from October 2018 to April 2019, providing 3,804 sets of wave measurements. Compared to the typical size of the NDBC buoys, $3 \mathrm{~m}$ in diameter, the Spotter is an order of magnitude smaller 
with a diameter of $40 \mathrm{~cm}$ so that it is more responsive to shorter waves. The Spotter buoy provides wave spectra over the range of $0.03-1 \mathrm{~Hz}$.

\subsection{Direct Estimates of Friction Velocity from Observed Wind Speeds}

To get empirical estimates for the coefficients $\beta$ and $\alpha$ in equations (2) and (4), we use the observed wind speed $U_{10}$ and wave spectral level $E_{0}$ to estimate $u_{*}$ directly. The friction velocity $u_{*}$ is related to surface wind stress as $\tau=\rho u_{*}^{2}$, and since $\tau$ is not observed directly by the NDBC buoys, we model it here.

While turbulent stresses dominate the momentum flux outside the wave-boundary layer, at the ocean surface, turbulent stresses are negligible due to the strong damping of the vertical turbulent wind speed component, so that the surface stress can can be represented as the sum of viscous stress $\tau_{v}$ and wave-induced stress $\tau_{w}$ as

$$
\tau=\tau_{v}+\tau_{w}
$$

For wind speed below $3 \mathrm{~m} / \mathrm{s}$ the viscous stress dominates, while the wave-induced stress (or form drag) is responsible for the majority of momentum exchange between the wind and the ocean surface for $U_{10} \gtrsim 7 \mathrm{~m} / \mathrm{s}$ (Donelan et al., 2006; Edson et al., 2013; Wu, 1969).

The viscous shear stress is modeled through a drag model, relating $\tau_{v}$ to the wind speed at $10 \mathrm{~m}$ above the sea surface $U_{10}$ by a viscous drag coefficient $C_{v}$ (Tsagareli et al., 2010) as

$$
\tau_{v}=\rho C_{v} U_{10}^{2}
$$

with

$$
C_{v}=-5 \times 10^{-5} U_{10}+1.1 \times 10^{-3} .
$$

All transfer of energy from the wind to the waves occurs through the wave-induced stress and is therefore determined through the wind input source function $S_{\text {in }}$ (e.g., see equation (1)):

$$
\tau_{w}=\rho g \int_{\omega} \frac{S_{i n}}{c(\omega)} \mathrm{d} \omega
$$

where $c(\omega)$ is phase speed of the wave propagation per radian frequency $\omega=2 \pi f$. The wind input source function is given by

$$
S_{\text {in }}(\omega)=\omega \gamma(\omega) E(\omega),
$$

with $\gamma(\omega)$ is the dimensionless temporal growth rate of wave energy. For $\gamma$, the parameterization of Donelan et al. (2006) is used:

$$
\gamma(\omega)= \begin{cases}G \sqrt{B_{n}}\left(\frac{U_{10}}{c(\omega)}-1\right)^{2} & \text { if } U_{10} / c(\omega) \geq 1 \\ 0 & \text { if } U_{10} / c(\omega)<1,\end{cases}
$$

where $G$ and $B_{n}$ are the sheltering coefficient and the spectral saturation, respectively. Note that conditions to $\gamma$ based on $U_{10} / c(\omega)$ were added to correct for physical inconsistencies in the original parameterization of $\gamma$ for low wind speeds (see Appendix A). Further, the spectral saturation and sheltering coefficient are given by (Donelan et al., 2006)

$$
\begin{gathered}
B_{n}(\omega)=\left(\omega^{5} E(\omega) / 2 g^{2}\right) A(\omega), \\
G=2.8-1.0\left\{1+\tanh \left[10 \sqrt{B_{n}}\left(\frac{U_{10}}{c(\omega)}-1\right)^{2}-11\right]\right\},
\end{gathered}
$$

where $A(\omega)=1.12\left(U_{10} / c_{p}\right)^{-0.5}\left(\omega / \omega_{p}\right)^{-0.95}+1 /(2 \pi)$ is the directional spreading function (Babanin \& Soloviev, 1998), $\omega_{p}$ is the peak radial frequency, and $c_{p}$ the phase speed at the peak frequency. This approach of modeling $\tau$ is similar to that used by Voermans et al. (2019) who showed that the modeled wind stress using Equations (5)-(12) compared well against direct observations of the wind stress, at least for wind speeds between 5 and $23 \mathrm{~m} / \mathrm{s}$. 


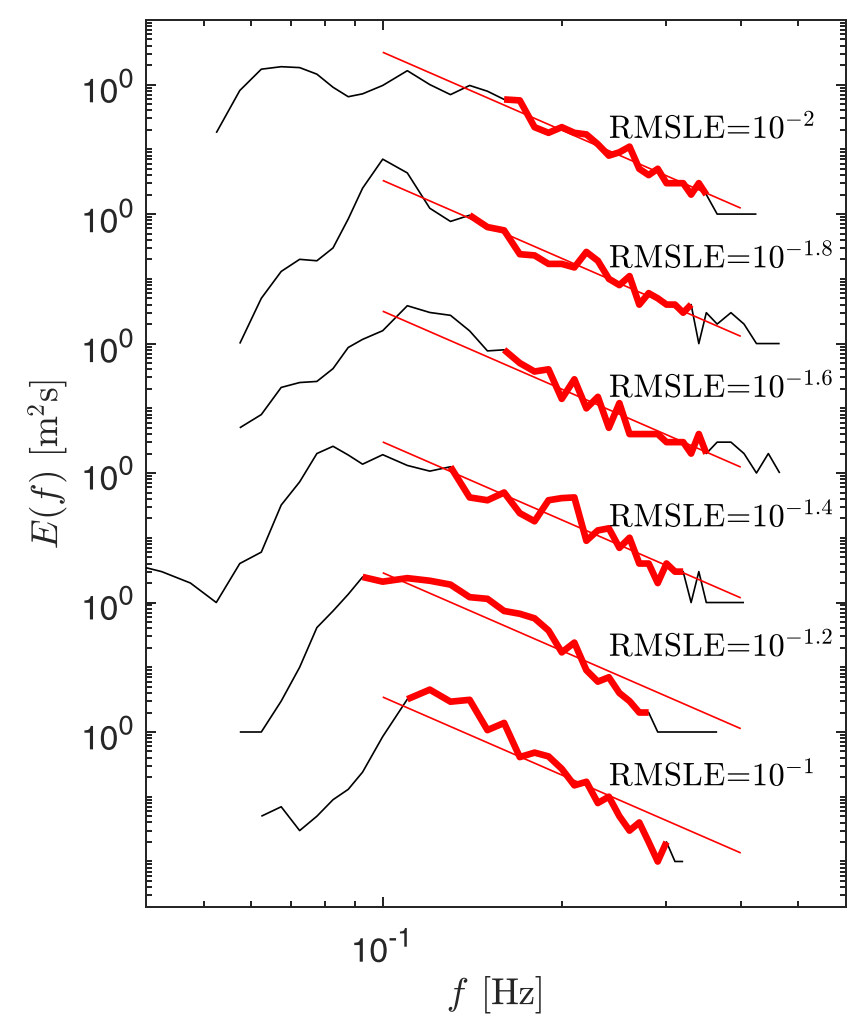

Figure 2. Examples of wave energy spectra for different values of the root-mean-square logarithmic error (RMSLE) of the $f^{-4}$ spectral fit. In this study, a threshold of RMSLE $=10^{-1.6}$ is chosen and any spectrum where RMSLE $>10^{-1.6}$ is not used hereafter.

As the wave-induced stress is strongly dependent on the upper limit of the measured high-frequency spectral tail, the unresolved wave energy at higher frequencies is modeled. Since we cannot identify a clear separation between the equilibrium range ( $f^{-4}$ slope) and the saturation range ( $f^{-} 5$ slope) in the majority of the wave energy spectra obtained by the NDBC buoys, we assume that the spectra are well described by a JONSWAP spectrum. A $f^{-5}$ slope is therefore fitted to the high-frequency end of the measured spectrum from $f=0.24$ to $f=0.485 \mathrm{~Hz}$. For the spectra derived from the NDBC buoy measurements, this means a spectral fit to 18 frequency bands. The unresolved high-frequency tail of the measured spectrum is then modeled to extend the measured spectrum from $f=0.485$ to $10 \mathrm{~Hz}$ (consistent with Tsagareli et al., 2010). Thus, if the wave energy spectrum and wind speed are known, both the viscous and wave-induced stress can be determined through equations (6)-(12). The modeled friction velocity is used to determine the Charnock parameter $\alpha$ through equations (3) and (4) and $\beta$ through equation (2).

\subsection{Wind Speed Estimate From Equilibrium Range}

To estimate the friction velocity using the generalized spectral form in the equilibrium range, a similar method was applied as used by Thomson et al. (2013). Equation (2) was fitted to a moving range of 20 consecutive frequency bands to determine $u_{*}$ from the fit with the lowest root-mean-square logarithmic error (RMSLE). The logarithmic error is nondimensional and is chosen to avoid bias toward the low frequencies of the fitting range. Consistent with Thomson et al. (2013), the frequency range with the lowest RMSLE typically spans $0.2<f<0.4 \mathrm{~Hz}$. Data were removed from further analysis when the RMSLE of the fit exceeded a certain threshold. Figure 2 presents six spectra falling into different error categories. Here a threshold of RMSLE $=10^{-1.6}$ was chosen and reduced the size of the data set by $25 \%$. As the generality of the physical $f^{-4}$ slope remains a topic of discussion, it is possible that by the removal of spectra with a deviating slope, physical aspects of wind-wave interactions may have been excluded unintentionally as well. However, in line with the scope of the study, our interests are only in those spectra, which present an equilibrium range with a $f^{-4}$ form. Using the values for $\beta$ estimated from observed wind speeds and the momentum balance in section 2.2, we use observed values of $E_{0}$ to estimate the friction velocity $u_{*}$. From $u_{*}$ and values for $\alpha$ derived from the model in section 2.2, the wind speed at $10 \mathrm{~m}$ elevation $\left(U_{10}\right)$ is estimated from equations (3) and (4). 


\subsection{Wind Direction Estimate From Equilibrium Range}

As the majority of energy transfer from wind to waves occurs at the high-frequency range of the wave energy spectrum, it seems reasonable to assume that the direction of wave propagation in this range closely approximates the wind direction. We use the lowest-order Fourier coefficients of the directional distribution to estimate the mean direction for each frequency (e.g., Kuik et al., 1988):

$$
\theta(f)=270^{\circ}-\frac{180^{\circ}}{\pi} \operatorname{atan} 2\left(\frac{b_{1}(f)}{a_{1}(f)}\right)
$$

where $a_{1}$ and $b_{1}$ are the Fourier coefficients. We then estimate the mean wave direction in the equilibrium range $\left(\theta_{e q}\right)$ as follows:

$$
\theta_{e q}=\operatorname{atan} 2\left(\frac{1}{N} \sum_{i=1}^{N} \sin \left(\theta_{f_{i}}\right), \frac{1}{N} \sum_{i=1}^{N} \cos \left(\theta_{f_{i}}\right)\right)
$$

where $N$ is the number of measured frequencies in the equilibrium range and the frequency components in the averaging of $\theta_{e q}$ are uniformly weighted. It should be noted that bimodal directional spreading can exist in the equilibrium range, particularly for frequencies larger than twice the peak frequency (Young et al., 1995). As the bimodal structure can be asymmetric in direction (relative to the wind direction) and in energy, the mean wave direction at a given frequency may not exactly follow the wind direction. These effects are likely higher order and not considered in this study.

\section{Results}

\subsection{Estimating $\beta$ and $\alpha$ From Observed Wind Speed and Wave Energy}

Assuming that the model for wind stress and friction velocity (i.e., following equations (5)-(12)) is reasonable, equation (2) can be used to estimate $\beta$ from observed wave energy levels.

The resulting estimates of $\beta$ are well sorted with wind speed, wave steepness (defined here as $H_{m 0} k_{p}$, where $k_{p}$ is the peak wave number corresponding to the peak period $\left.T_{p}\right)$, and wave age $\left(U_{10} / c_{p}\right.$, where $c_{p}$ is the phase speed corresponding to $T_{p}$ ), as shown in Figure 3. The dependence of $\beta$ on wave age can also be interpreted as the influence of swell on the energy level of the sea state (Vincent et al., 2019). We did not find any such dependency to a variety of other dimensional and nondimensional wind and wave parameters.

For $U_{10}>6 \mathrm{~m} / \mathrm{s}, \beta$ can be reasonably approximated by a constant value of about 0.009 , with a weak decrease of $\beta$ observed for larger wave ages. For $U_{10}<6 \mathrm{~m} / \mathrm{s}$, the variance in estimates for $\beta$ increases. Overall, the observed values of $\beta$ in this study correspond well to those referred to by Phillips (1985), with $\beta=$ $[0.006-0.011]$ and found by Juszko et al. (1995) with $\beta=0.0122 \pm 3.6 \times 10^{-3}$.

From the modeled values of $u_{*}$ (equations (5)-(12)) and assuming the presence of a logarithmic wind profile above the ocean surface (equation (3)), the Charnock parameter $\alpha$ is approximated from the estimated roughness length $z_{0}$ (equation (4)) using only the rough surface term; that is, $z_{0}=\alpha u_{*}^{2} / g$. The results for $\alpha$ are compared against wave steepness and wave age (Figures $4 \mathrm{a}$ and $4 \mathrm{~b}$, respectively). Estimates of $\alpha$ are $O(0.01)$ and similar to the those observed and adopted by others (e.g., Johnson et al., 1998; Thomson et al., 2013; Wu, 1969). However, weak trends of $\alpha$ with wave steepness and wave age are observed and a best fit to the data yields

$$
\begin{gathered}
\alpha=0.06\left(H_{m 0} k_{p}\right)^{0.70}, \\
\alpha=0.14\left(\frac{u_{*}}{c_{p}}\right)^{0.61}
\end{gathered}
$$

These formulations for $\alpha$ provide a slightly better fit to the data set used in this study than those determined by Edson et al. (2013) (see Figure 4 for comparison). The convergence of $\alpha$ for small wave age signifies the change of the ocean surface roughness to a smooth roughness regime. It is worth mentioning that a multi variable correlation between $\alpha$, wave steepness and wave age is not much larger than the cross correlation between $\alpha$ and wave steepness or wave age, implying that estimates of $z_{0}$ are unlikely to improve using a multivariable parameterization of $\alpha$. 

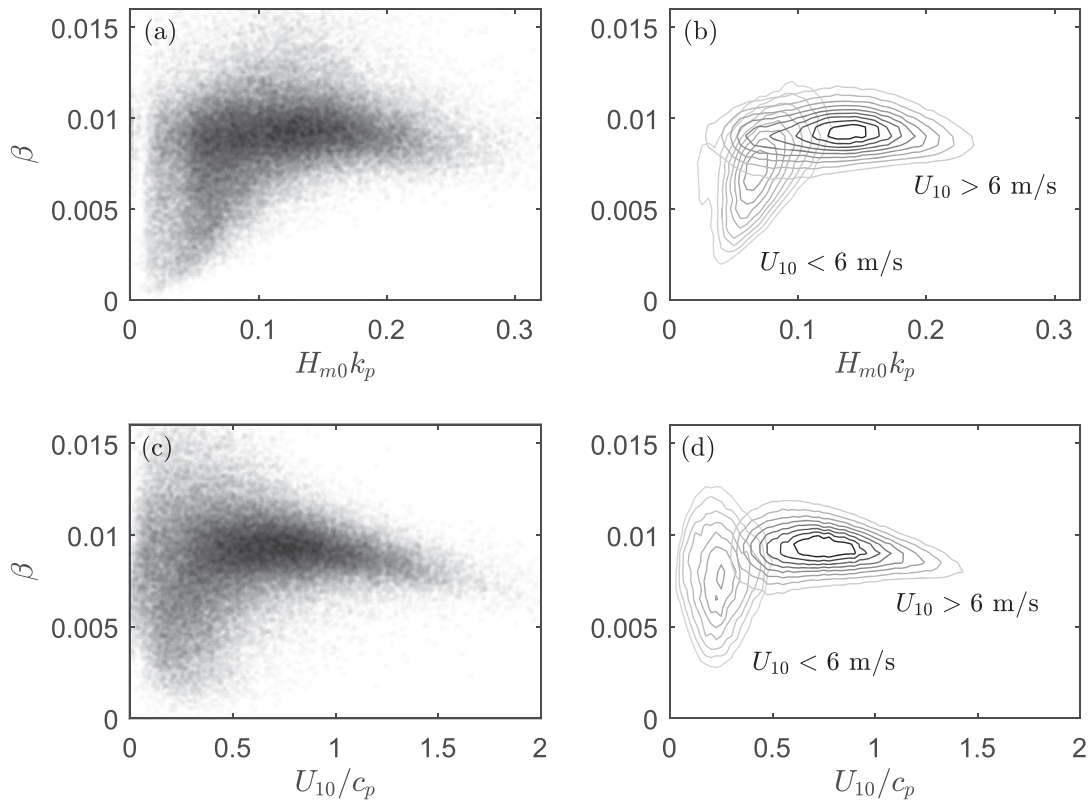

Figure 3. Variation of the $\beta$ with (a, b) mean wave steepness and (c, d) mean wave age. $\beta$ is obtained through equation (2) by modeling the friction velocity $u_{*}$ (equation (5)-(12)). In (b) and (d) density contours are provided, distinguishing between values of $\beta$ for $U_{10}<6$ and $U_{10}>6 \mathrm{~m} / \mathrm{s}$.

With an expression for $\alpha$, the modeled wind stress can be used to model the wind speed (equations (3) and (4); note that this model uses a priori knowledge of the wind speed) and can be compared against the measured wind speed (Figure 5). For mild winds, that is, $U_{10}<5 \mathrm{~m} / \mathrm{s}$, the modeled wind speed compares well to the measured wind speed. With increasing wind speed, the error of the modeled wind speed increases steadily. The close fit observed between the modeled and measured wind speed (i.e., the root-mean-square error, RMSE, is $0.36 \mathrm{~m} / \mathrm{s}$ for the time series shown in Figure 5) is supportive of the methodology used to model the wind stress and thus the parameterizations of $\beta$ and $\alpha$.

A comparison of the modeled and measured wind speed of the complete data set for $\alpha=0.012$, and the wave steepness dependent $\alpha$ (equation (15)) suggests that the use of a constant value of the Charnock parameter is reasonable as a first estimate (Figure S1 in the supporting information). However, improvement of the modeled wind speed can be achieved using the wave steepness or wave age-dependent parameterizations of $\alpha$, for example, equations (15) and (16) (Figure S2).

\subsection{Estimating Wind Speed Directly From Wave Observations}

With calibrated formulations for $\beta$ and $\alpha$, the wind speed is estimated based on the properties of wave energy spectrum alone (equations (2)-(4)). For $\alpha$, we choose the wave steepness-dependent Charnock parameter (Equation (15)), as errors in $u_{*}$ will propagate further into the estimate of $U_{10}$ through the wave
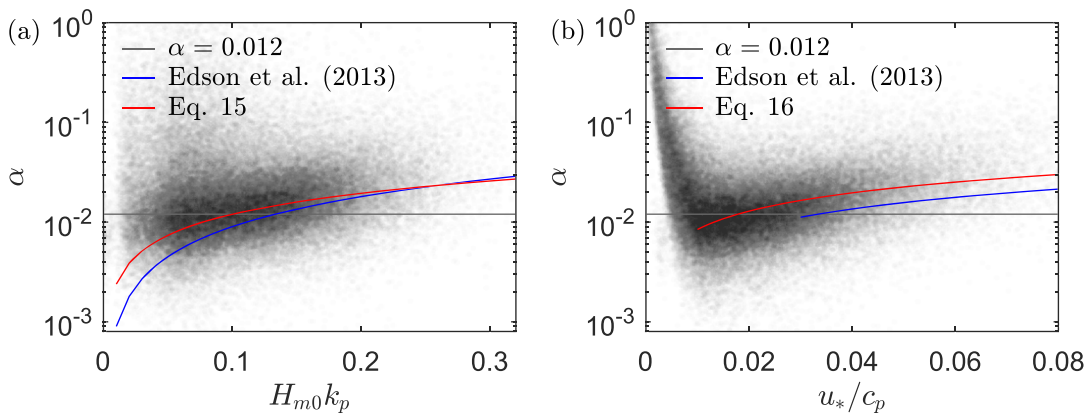

Figure 4. Variation of the Charnock parameter $\alpha$ with (a) peak frequency wave steepness and (b) wave age, where $\alpha$ is obtained through equations (3) and (4) by modeling the wind stress using equation (5)-(12). Curve fit to the data is given in red; formulations of $\alpha$ given by Edson et al. (2013) are given in blue. 


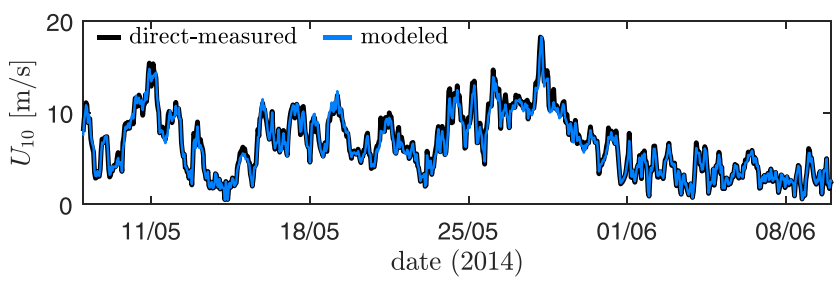

Figure 5. Time series comparison of the measured (black) and modeled (blue) wind speed of NDBC 46026 in 2014. Note that the model uses a priori knowledge of the wind speed. The RMSE between the measured and modeled time series is $0.36 \mathrm{~m} / \mathrm{s}$.

age-dependent parameterization of $\alpha$. An example of direct-measured and proxy-measured wind speed time series is shown in Figure 6a, where the proxy-measured wind speed of the shown time series has a RMSE $=1.7 \mathrm{~m} / \mathrm{s}$. The trend and magnitude of the wind speed are generally approximated well, though for wind speeds below about $7 \mathrm{~m} / \mathrm{s}$ the relative error becomes quite large. A reoccurring feature of error in the proxy-measured wind speed is the strong delay of the estimate when $U_{10}$ drops from a moderate to mild wind speed and increases again shortly after. In the example presented, this is most notable around 14-15 May where it takes nearly 1 day before the proxy-measured wind speed is following the direct-measured wind trend and magnitude again. To a lesser extent, this can be seen around 9 and 24 May as well.

Comparison of the complete data set of the proxy-measured and direct-measured wind speed suggests that the wind speed can be reasonably estimated using wave properties only (Figure 7a). The RMSE for wind speeds between 3 and $12 \mathrm{~m} / \mathrm{s}$ is about $2 \mathrm{~m} / \mathrm{s}$. Beyond $U_{10}=12 \mathrm{~m} / \mathrm{s}$, the absolute error increases steadily but remains about $17 \%$ of the actual measured wind speed (Figure $7 \mathrm{~b}$ ). The bias is small for moderate wind speeds but increases with wind speed for $U_{10}>14 \mathrm{~m} / \mathrm{s}$. The accuracy of the proxy-measured wind speed is therefore comparable to that of other indirect methods (for example, 1.0-1.7 m/s for satellite radar altimeters, Zieger et al., 2009).

\subsection{Estimating Wind Direction Directly From Wave Measurements}

Time series of the measured $\left(\theta_{\text {wind }}\right)$ and estimated wind direction $\left(\theta_{e q}\right)$ are shown in Figure 6b. Good correspondence between measurements and estimates are observed for moderate to high wind speeds (RMSE $=$ $16^{\circ}$ for $U_{10}>7 \mathrm{~m} / \mathrm{s}$ ); however, large errors are seen for the proxy-measured wind direction during low wind speeds (RMSE $=57^{\circ}$ for $U_{10}<7 \mathrm{~m} / \mathrm{s}$ ). This is consistent with the comparison of $\theta_{\text {wind }}$ against $\theta_{e q}$ for the complete data set (Figure 8), where the RMSE $<20^{\circ}$ for $U_{10}>11 \mathrm{~m} / \mathrm{s}$. The accuracy of the estimated wind direction has a noticeable dependency on the absolute fetch (fetch is here defined as the distance between the coast and the buoy in the direction of the mean wind but is capped at an arbitrary maximum of 3,000 km), where the error tends to increase when fetches become shorter. For observations with fetch
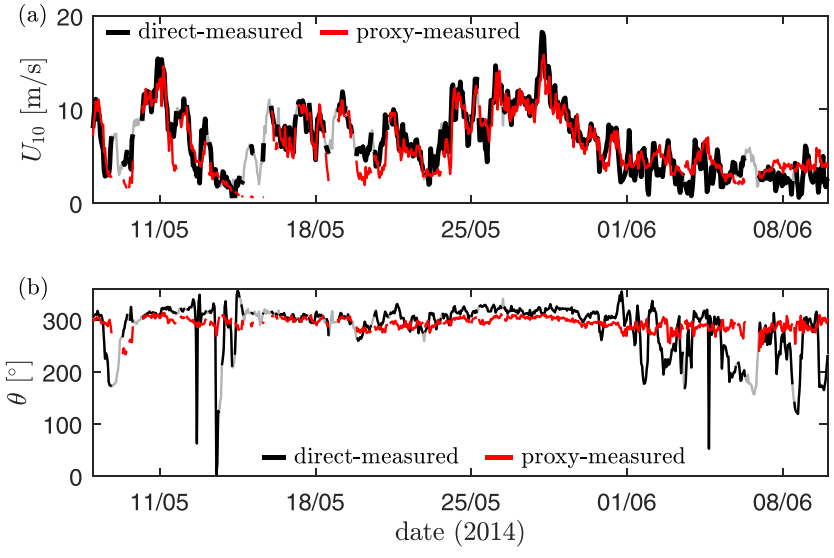

Figure 6. Time series comparison of the direct-measured (black) and proxy-measured (red) wind speed (a) and wind direction (b) of NDBC 46026 in 2014. The RMSE of the proxy-measured wind speed and direction are $1.7 \mathrm{~m} / \mathrm{s}$ and $42^{\circ}$, respectively. The sections of the direct-measured wind properties in gray correspond to the instances where estimates of wind properties are unavailable due to quality control reasons. 

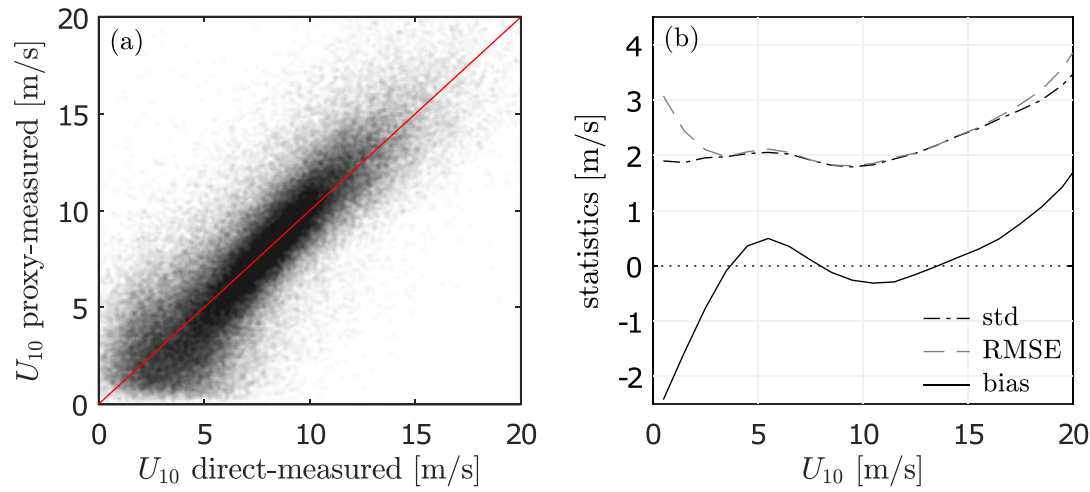

Figure 7. (a) Comparison of the proxy-measured wind speed based on the equilibrium range of the wave energy spectrum against direct-measured wind speed. (b) The bias, standard deviation (std), and RMSE of the proxy-measured wind speed.

larger than $100 \mathrm{~km}$, the RMSE is smaller than $20^{\circ}$ for wind speeds between 10 and $20 \mathrm{~m} / \mathrm{s}$. These dependencies are similar to those observed by Pickett et al. (2003) based on QuikSCAT satellite data, where the RMSE of the estimated wind direction was found to be $15^{\circ}$ for wind speeds above $6 \mathrm{~m} / \mathrm{s}$ and observations $500 \mathrm{~km}$ offshore and $26^{\circ}$ for wind speeds above $6 \mathrm{~m} / \mathrm{s}$ and not further than $41 \mathrm{~km}$ from the coastline.

\section{Discussion}

Sources of error in the estimated wind speed based on the equilibrium range of the wave spectrum may be related to (i) inaccuracies in the physical processes underlying the spectral energy balance in the equilibrium range, (ii) the parameterizations of coefficients $\alpha$ and $\beta$, (iii) buoy specifications, including the accuracy of the wind and wave measurements and buoy properties, and (iv) validity of the equilibrium assumption in the equilibrium range.

During very low wind speeds, that is, $U_{10}<4 \mathrm{~m} / \mathrm{s}$, the shift in physical exchange processes between wind and waves likely dominates the error. The exchange of momentum between the atmosphere and the ocean surface during mild winds is driven by viscous drag (e.g., and Figure Alb; Kudryavtsev \& Makin, 2001) rather than form drag such that most of the momentum is transferred to the surface currents rather than the waves. Hence, it is unlikely that the wind stress in such cases can be determined from wave measurements alone.

For more energetic sea states, increases in bias are observed for $U_{10} \gtrsim 13 \mathrm{~m} / \mathrm{s}$ (Figure $7 \mathrm{~b}$ ), similar to the onset of bias noted by Thomson et al. (2013) at $15 \mathrm{~m} / \mathrm{s}$, who argued that this could be attributed to wave modulation in the equilibrium range by swell, consistent with the observations of Vincent et al. (2019). Alternatively, it could be argued that the physical processes that lead to wave breaking start to change, such that the energy balance in the equilibrium range originally proposed by Phillips (1985), do not necessarily hold anymore.
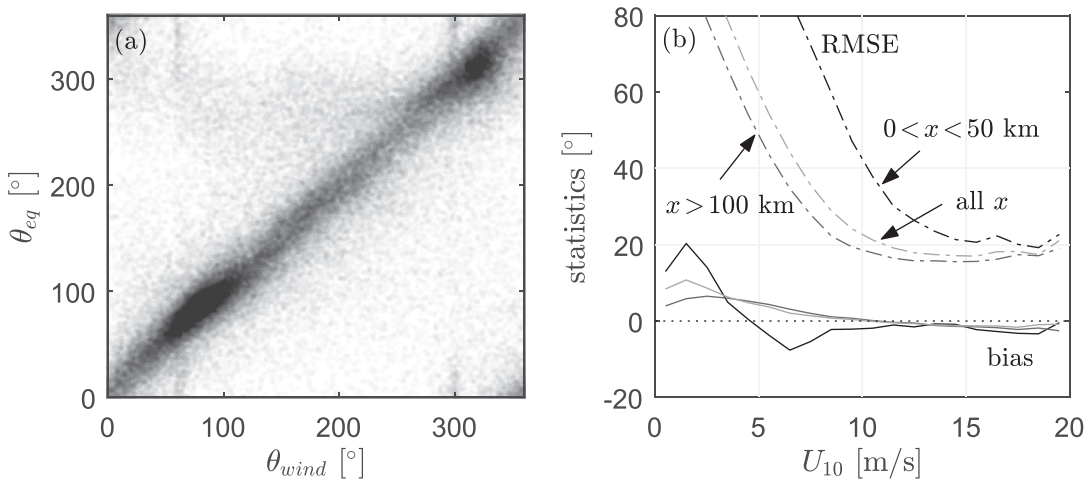

Figure 8. (a) Comparison of proxy-measured $\left(\theta_{e q}\right)$ and direct-measured wind direction $\left(\theta_{\text {wind }}\right)$ and (b) bias (solid line) and RMSE (dash-dotted line) of the proxy-measured wind direction with fetch (black for fetches between 0 and $50 \mathrm{~km}$, dark gray for fetches larger than $100 \mathrm{~km}$, and light gray for all fetches). 

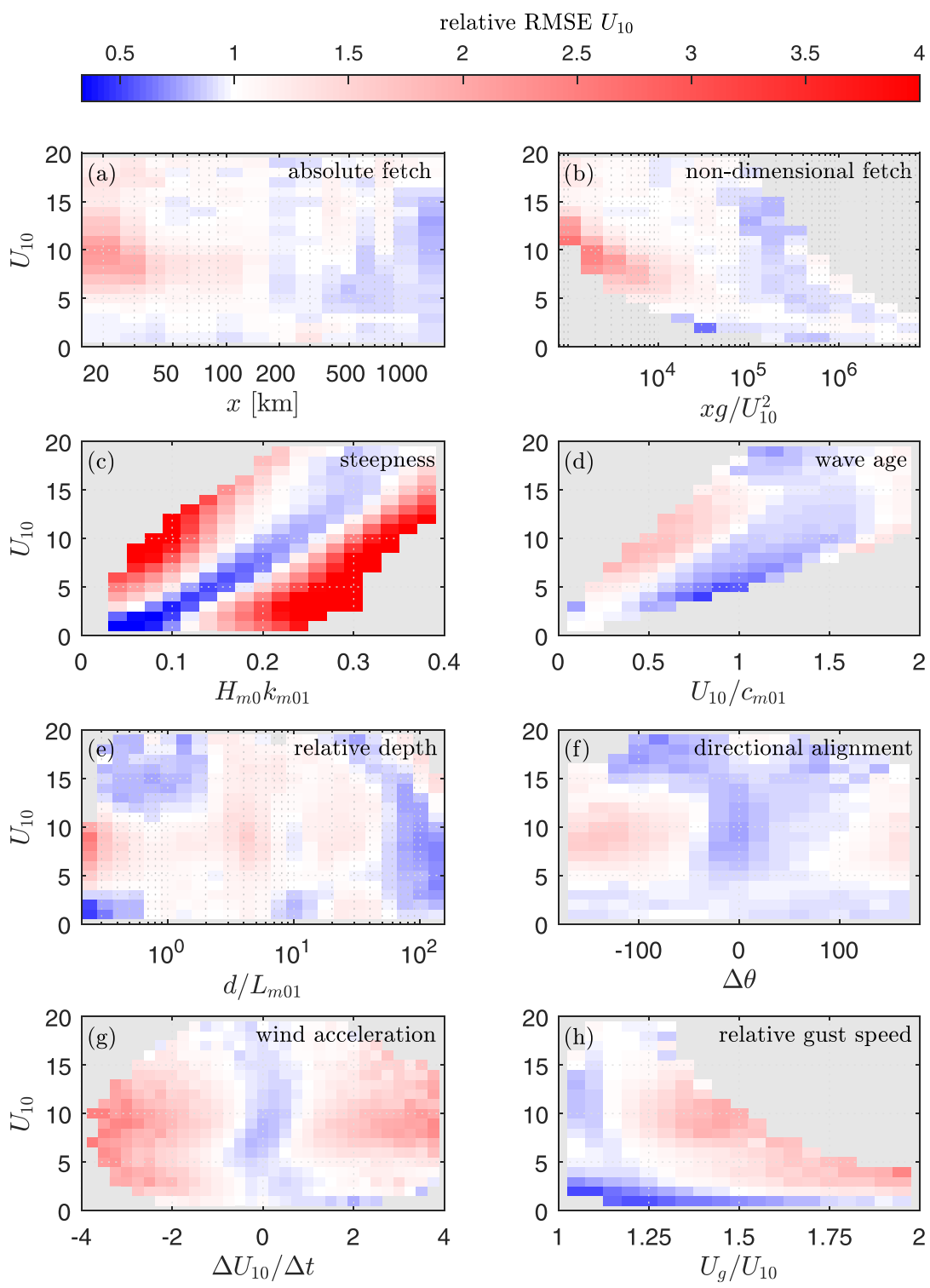

Figure 9. Relative error of the proxy-measured wind speed with (a) absolute fetch $x$, (b) nondimensional fetch $x g / U_{10}^{2}$, (c) mean wave steepness $H_{m 0} k_{m 01}$, (d) mean wave age $U_{10} / c_{m 01}$, (e) relative depth $d / L_{m 01}$, (f) directional alignment between the peak wave and wind $\Delta \theta$, (g) hourly wind acceleration $\Delta U_{10} / \Delta t$, and (h) relative gust speed $U_{g} / U_{10}$. The relative error is determined by the ratio of the RMSE per bin to the RMSE per wind speed.

Part of the underestimation of the wind speed for $U_{10}>13 \mathrm{~m} / \mathrm{s}$ is methodological, as the anomemeter height (typically at a height of 4-5 m above mean sea level) becomes similar in order of magnitude as the significant wave height of the sea state, and therefore, wind speed measurements are within the wave boundary layer. Extrapolation of these wind speed measurements to a standard height of $10 \mathrm{~m}$ based on a logarithmic wind speed profile will then lead to an overestimation of the actual wind speed (Babanin et al., 2018).

\subsection{Sensitivity Analysis}

In Figure 9, the relative RMSE (i.e., the RMSE of each bin normalized by the RMSE for each wind speed, see the Acknowledgments section for the absolute RMSE) of the proxy-measured wind speed is related to various wind and wave parameters, namely, fetch (both dimensional $x$ and nondimensional $x g / U_{10}^{2}$ ), wave steepness ( $H_{m 0} k_{m 01}$, where $k_{m 01}$ is the wave number corresponding to the mean period $\left.T_{m 01}\right)$, wave age $\left(U_{10} / c_{m 01}\right.$, with phase speed $c_{m 01}=L_{m 01} / T_{m 01}$ and wave length $\left.L_{m 01}\right)$, relative depth $\left(d / L_{m 01}\right.$, where $d$ is the water depth), directional alignment of the peak frequency wave direction to the wind $(\Delta \theta)$, wind acceleration $\left(\Delta U_{10} / \Delta t\right.$, where $\Delta t=1 \mathrm{hr})$, and the relative gust speed $\left(U_{g} / U_{10}\right.$, where $U_{g}$ is the gust speed). Most noticeable source 

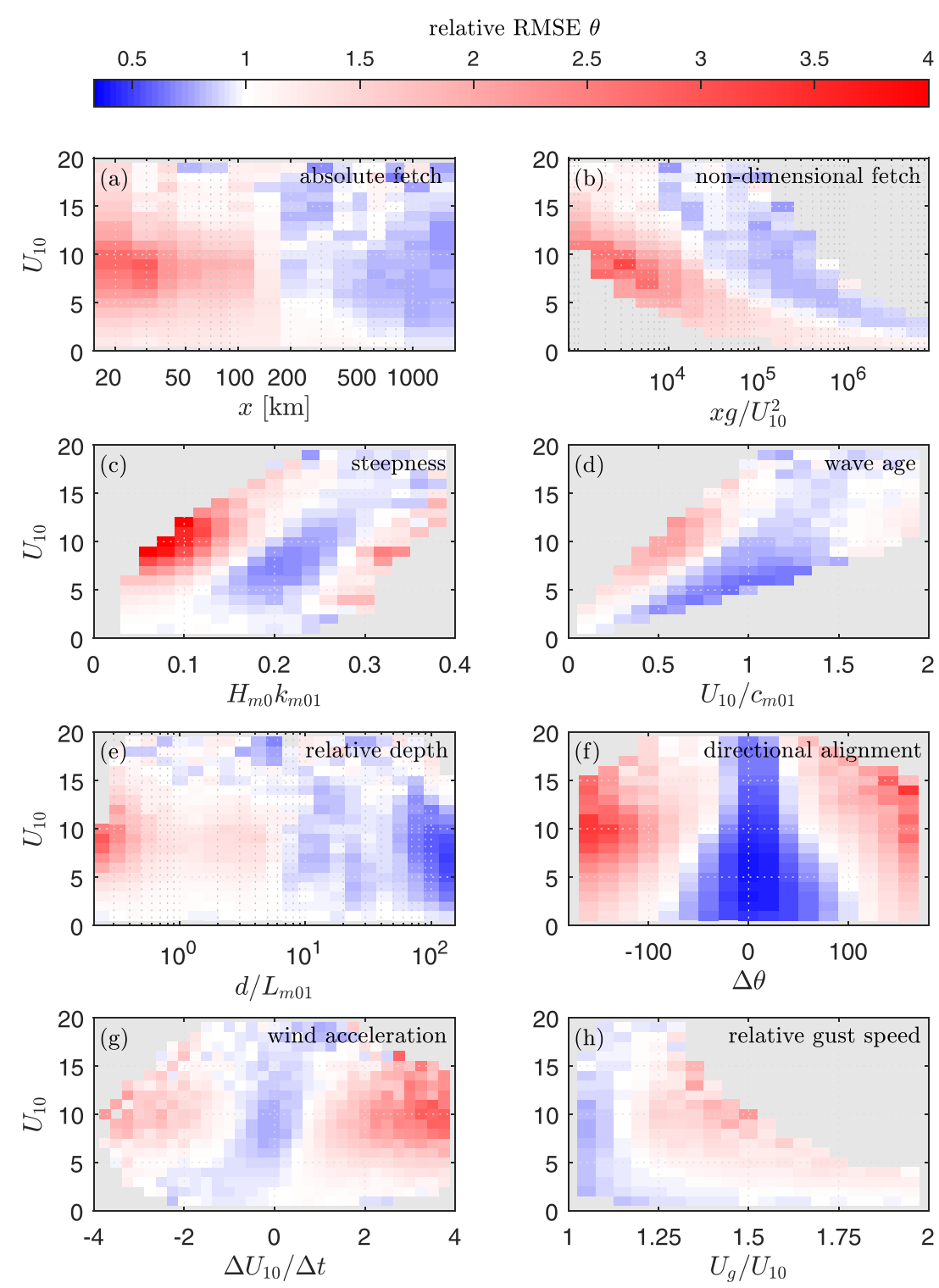

Figure 10. Relative error of the proxy-measured wind direction with (a) absolute fetch $x$, (b) nondimensional fetch $x g / U_{10}^{2}$, (c) mean wave steepness $H_{m 0} k_{m 01}$, (d) mean wave age $U_{10} / c_{m 01}$, (e) relative depth $d / L_{m 01}$, (f) directional alignment between the peak wave and wind $\Delta \theta$, (g) hourly wind acceleration $\Delta U_{10} / \Delta t$, and (h) relative gust speed $U_{g} / U_{10}$. The relative error is determined by the ratio of the RMSE per bin to the RMSE per wind speed.

of scatter is the strong relative bias induced by the mean wave steepness (Figure 9c) where the line of lowest relative RMSE corresponds closely to the bin average of $H_{m 0} k_{m 01}$ per wind speed (not shown here). It should be mentioned that the observed relation between the wave steepness and the relative RMSE is not a consequence of the steepness-dependent parameterization of $\alpha$ used here as similar results of the relative RMSE were observed when using $\alpha=0.012$. However, this strong dependence could be a consequence of imposing a $f^{-4}$ spectral slope to the measured spectra. Short fetches (Figures 9a and 9b), large misalignment between wind and peak wave direction (Figure 9f) and strong variability in mean and instantaneous wind speed (Figures 9g-9h) also contribute to the observed discrepancy between the direct-measured and proxy-measured wind speed, presumably as spatial and temporal gradients of spectral energy cannot be ignored anymore. The error associated with directional misalignment of the peak wave and the wind could indicate instances where swell is important, which is known to influence the momentum flux to and from the wind (García-Nava et al., 2009), whereas short- and long-term variability of the wind speed are found to cause variability in $z_{0}$ and $\beta$, respectively (Toba \& Ebuchi, 1991; Toba et al., 1988). 


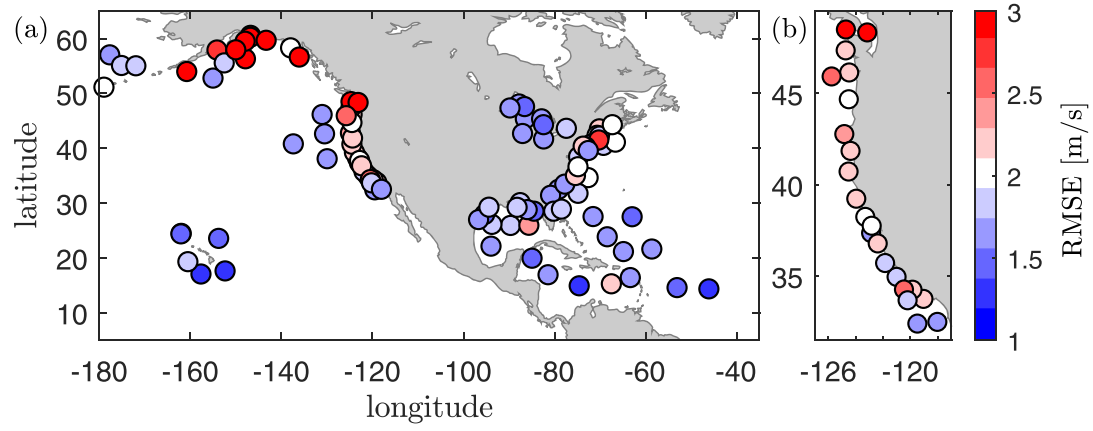

Figure 11. RMSE of the proxy-measured wind speed for wind speeds between 5 and $15 \mathrm{~m} / \mathrm{s}$ for the individual NDBC buoys in (a) American continent (b) and the West Coast of the United States. The colour axis of the RMSE is capped at $3 \mathrm{~m} / \mathrm{s}$, where the largest RMSE observed is $4.4 \mathrm{~m} / \mathrm{s}$. As the average RMSE for $5<U_{10}<15 \mathrm{~m} / \mathrm{s}$ for the complete data set is $2 \mathrm{~m} / \mathrm{s}$, blue and red marked buoys indicate below and above average errors for the proxy-measured wind speed, respectively.

Similarly, the relative RMSE of the estimated wind direction is shown in Figure 10. Strongest dependence of the error is observed with the directional alignment between the wind direction and the wave direction of the peak frequency (Figure 10f), which might suggest that swell interacts with the short waves in the equilibrium range (Vincent et al., 2019). As seen in Figures 8b and 10a, the error of the proxy-measured wind direction is sensitive to the absolute fetch. The observed dependence between the relative RMSE and the relative depth is likely a consequence of the geophysical correlation between local fetch and water depth (Figure 10e). Non stationarity of the wind (i.e., hourly wind acceleration and relative gust speed) tends to increase the RMSE of the proxy-measured wind direction (Figures $10 \mathrm{~g}$ and $10 \mathrm{~h}$ ).

To estimate the error in wind speed $\Delta U_{10}$ (and the normalized error $\left.\epsilon=\left(U_{10}+\Delta U_{10}\right) / U_{10}\right)$ associated with the parameterizations of $\beta$ and $\alpha$, the 25th and 75th percentiles of $\beta$ and $\alpha$ (e.g., see Figures 3 and 4, respectively) were determined. The variability of $\beta$ leads to an error of $0.49<\epsilon<1.06$ for $5<U_{10}<$ $8 \mathrm{~m} / \mathrm{s}$, and $0.82<\epsilon<1.08$ for $8<U_{10}<20 \mathrm{~m} / \mathrm{s}$. Similarly, the variability in $\alpha$ leads to an error in wind speed of $0.91<\epsilon<1.06$ for $5<U_{10}<20 \mathrm{~m} / \mathrm{s}$. This suggests that part of the error between the measured and estimated wind speed is associated with the parameterization of $\alpha$ and $\beta$. It should be noted, however, that spurious errors in the estimated values of $\alpha$ and $\beta$ can persist through methodological errors in the modeling of the wind stress, that is, the extrapolation of the measured high-frequency tail to model the wind stress and the calibration of $\alpha$ and $\beta$ thereof. However, as the parameterizations of $\beta$ and $\alpha$ are within the empirical range as observed by others, and the estimated wind speed based on the modeled wind stress through equations (5)-(12) corresponds well to the measured wind speed (Figure S1), we expect the contribution of methodological errors to the variability $\beta$ and $\alpha$ to be small.

\subsection{Variability Between Sites}

The accuracy of the proxy-measured wind speed can vary significantly with deployment sites (i.e., Figure 11). When the RMSE of the proxy-measured wind speed for $5<U_{10}<15 \mathrm{~m} / \mathrm{s}$ for each NDBC buoy is considered, the smallest and largest RMSE observed are 1.2 and $4.4 \mathrm{~m} / \mathrm{s}$, respectively. Differences in the physical properties of the NDBC buoys are unlikely to be a source of errors in wind speed estimates as no correlation is observed between buoy properties (i.e., buoy diameter, material, and sensor used) and the RMSE. In particular, while the size of the buoy is a critical property of the buoy in resolving the high-frequency tail of the spectrum, $96 \%$ of the NDBC buoys considered in this study have a diameter of $3 \mathrm{~m}$. Thus, environmental and geophysical conditions specific to the sites of the buoy are more likely to cause such differences between the buoys. Significant errors are seen for buoys located in the northern part of the West Coast, where some buoys have a RMSE $=3-4 \mathrm{~m} / \mathrm{s}$. While the buoys with below average RMSE are typically located further offshore, the absolute distance of the buoy to the coastline does not seem to be a determining factor, as the wind estimates based on measurements from NDBC buoys located in the Great Lakes are better than the average NDBC buoy proxy measurements. Close inspection of the buoys in the Great Lakes shows that bias starts to increase considerably for $U_{10} \gtrsim 12 \mathrm{~m} / \mathrm{s}$, an error that could be explained by a small nondimensional fetch. The cluster of NDBC buoys near Alaska with the largest observed RMSE does not show large scatter (or standard deviation) of the wind speed estimates but does reveal very large bias that leads to this 

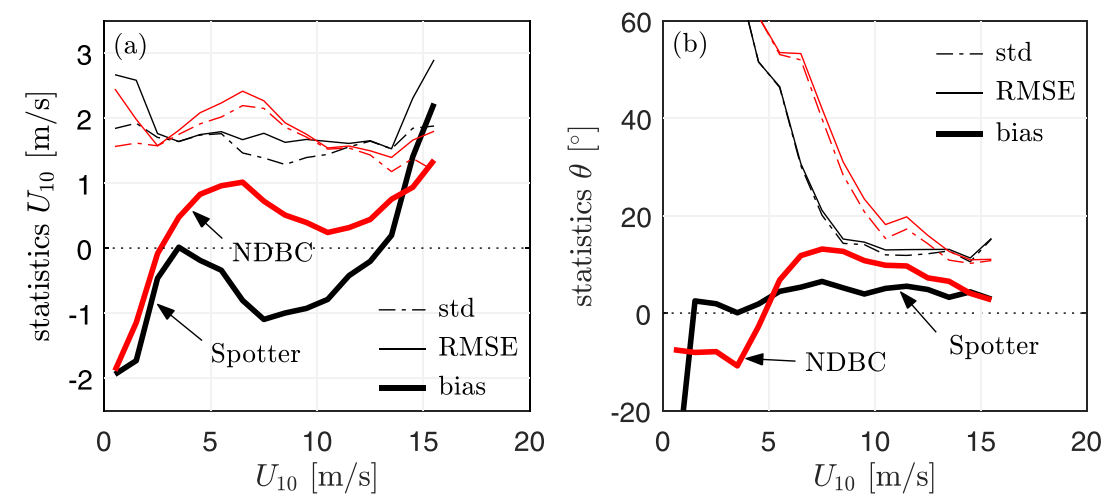

Figure 12. Comparison of the proxy-measured and direct-measured wind speed (a) and wind direction (b) for a Spotter (black) and NDBC buoy (red). The Spotter was deployed next to NDBC 46026 between October 2018 and April 2019. The bias is given by the thick solid line, the RMSE by the thin solid line, and the standard deviation by the broken line.

large RMSE. The variability between buoys could be a result of the orientation of the dominant wind direction between the different sites, where limited fetch seems to increase the error (e.g., see Figures 9a and $9 b$ ), whereas winds parallel to the coast are argued to attain a more complex balance between energy source terms (Long \& Resio, 2007).

\subsection{Influence of Buoy Size}

Relative errors of the proxy-measured wind speed and wind direction are largest for low wind sea states where the relative error steadily increases with decreasing wind speed for $U_{10}<9 \mathrm{~m} / \mathrm{s}$ (e.g., Figure 7b). Part of this error is caused by the vast size of the NDBC buoys, which limits the measurement accuracy of high-frequency waves and small-amplitude waves and is therefore most noticeable during milder winds when the wind wave energy is lowest. The impact of the buoy size on the accuracy of the wind estimates is shown in Figure 12 where proxy measurements of wind speed and direction are compared for a 40-cm-diameter Spotter buoy (Sofar Ocean Technologies) deployed adjacent to the 3-m-diameter NDBC buoy 46026 (Figure 12). For very low winds $\left(U_{10}<4 \mathrm{~m} / \mathrm{s}\right.$ ) and moderate to high winds (about $U_{10}>$ $10 \mathrm{~m} / \mathrm{s}$ ), the standard deviation and RMSE of the estimated wind speed and direction is comparable for both buoys. However, for wind speeds between 4 and $9 \mathrm{~m} / \mathrm{s}$, estimates of wind speed and direction based on the wave measurements of the Spotter buoy show an average improvement of $0.5 \mathrm{~m} / \mathrm{s}$ for the estimated wind speed and $15^{\circ}$ for the wind direction. This is a substantial improvement considering that the RMSE of the proxy-measured wind speed based on the wave measurements of the NDBC buoys is about $2 \mathrm{~m} / \mathrm{s}$ in this wind speed range. This improvement would suggest that smaller wave buoys are able to resolve the high-frequency end of the spectrum more accurately (Figure 13). Note that further improvement could be obtained by removing the bias from the proxy-measured wind properties. However, this requires further studies into the effect of form factor of the Spotter buoy.

\section{Concluding Remarks}

Collocated and concurrent observations of surface winds and waves enhance the value of meteorological in situ observations as wind-generated waves are, fundamentally, generated by wind. Collocated observations of wind and waves are, however, often not available. The purpose of this paper is to test a method to estimate wind measurements indirectly by small-size wave buoys, without the use of an anemometer mast. The accuracy of the estimated wind speed (RMSE $=2 \mathrm{~m} / \mathrm{s}$ for $3<U_{10}<12 \mathrm{~m} / \mathrm{s}$ and a relative error of approximately $17 \%$ for $10<U_{10}<20 \mathrm{~m} / \mathrm{s}$ ) and direction (RMSE of up to $20^{\circ}$ for $10<U_{10}<20 \mathrm{~m} / \mathrm{s}$ ) based on the shape of the wave energy spectrum are found to be comparable to observations through satellite altimetry and of the same order as the accuracy of the directly measured wind properties. Wave buoy systems can therefore provide a suitable proxy for the surface winds without the need of an anemometer. Once implemented, such wave-buoy capability can expand the coverage of collocated wind and wave observations to assist metocean models and augment existing coastal and oceanic monitoring systems. 


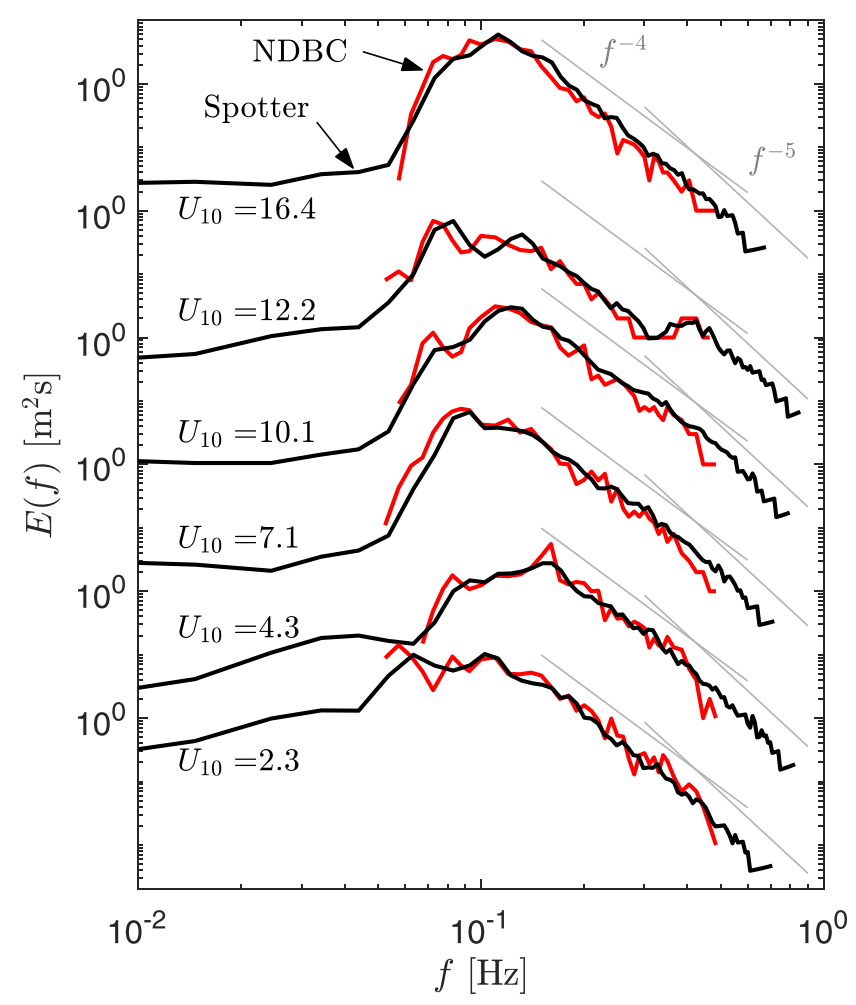

Figure 13. Comparison of wave energy spectra of the colocated Spotter (black) and NDBC Buoy 46026 (red) for different wind speed.

The estimation of wind properties can be improved by reducing the dimensions of the buoy as smaller wave buoys are more likely to resolve the high-frequency end of the spectrum accurately. For the studied deployment site, the RMSE of the estimated wind speed and direction of a Spotter wave buoy improves on average by $0.5 \mathrm{~m} / \mathrm{s}$ and $15^{\circ}$, respectively, for wind speeds between 4 and $9 \mathrm{~m} / \mathrm{s}$. Though the parameterizations of $\beta$ and $\alpha$ explain part of the error observed, different parameterizations of $\alpha$ have only limited impact on the accuracy of wind speed estimates. It therefore seems that further improvement of wind properties using wave observations relies on an improved understanding of the balancing spectral energy terms that lead to the spectral shape in the equilibrium range. For the time being, improvement can be achieved by applying more strict selection criteria to the data. For example, the RMSE can be reduced by using a threshold for both the misalignment between the direction of the (peak) wave and the mean wave direction in the equilibrium range and the absolute fetch. To determine the impact of selection criteria on the RMSE of the data set used in this study, the reader is referred to Text S1 in the supporting information.

Another approach to estimate the wind speed indirectly is by following a momentum balance rather than energy balance. In particular, when the wave spectrum is known, the only unknown in the modeling of the wind stress (as the sum of the viscous and wave-induced stress) is the wind speed. Unfortunately, the dependence of the wave-induced stress on wind speed is highly nonlinear and therefore sensitive to small errors in wind speed. Moreover, during mild winds, the viscous stress dominates the wind stress which makes it impossible to predict wind speed on wave measurements alone following this approach.

\section{Appendix A: Correction to Wave-Induced Stress Parameterization}

The wave growth function as given by Donelan et al. (2006) is:

$$
\gamma(\omega)=G \sqrt{B_{n}}\left(\frac{U_{10}}{c(\omega)}-1\right)^{2}
$$

For low wind speeds, this parameterization leads to physically incorrect trends of $\tau_{w}$, as the wave-induced stress appears to increase with decreasing wind speed for $U_{10} \lesssim 4 \mathrm{~m} / \mathrm{s}$ and dominates over the viscous stress 

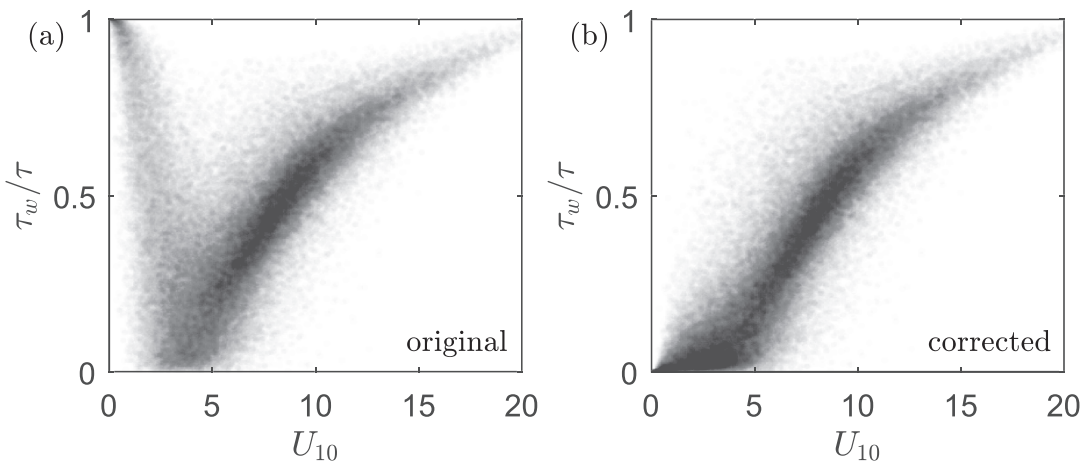

Figure A1. The contribution of the wave-induced stress $\left(\tau_{w}\right)$ to the wind stress $(\tau)$ consisting of the sum of the wave-induced stress and viscous stress for (a) the original parameterization of $\gamma$ (equation (A1)) and (b) a low wind speed correction for $\gamma$ given by equation (10). Note that $1-\tau_{w} / \tau$ is the fractional contribution of the viscous shear stress to the wind stress at the ocean surface.

(Figure A1a). This physical inconsistency is caused by the parabolic term $\left(U_{10} / c(\omega)-1\right)^{2}$ in the wave growth function $(\gamma)$. Equation (A1) is therefore altered by forcing $\gamma$ to 0 at any frequency where $U_{10} / c(\omega)<1$; that is, it assumes that there is no transfer of energy between wind and waves when waves travel faster than the wind. For convenience, the corrected equation (equation (10)) is repeated below:

$$
\gamma(\omega)=\left\{\begin{array}{c}
G \sqrt{B_{n}}\left(\frac{U_{10}}{c(\omega)}-1\right)^{2} \text { if } U_{10} / c(\omega) \geq 1 \\
0 \text { if } U_{10} / c(\omega)<1 .
\end{array}\right.
$$

This correction leads to a physically correct partitioning of the $\tau_{w}$ and $\tau_{v}$ for low wind speeds (Figure A1b), that is, a monotonic decrease of $\tau_{w} / \tau$ for decreasing wind speed.

\section{Acknowledgments}

J. J. V. and A. V. B. were supported by the DISI Australia-China Centre through Grant ACSRF48199. A. V. B. acknowledges support from the U.S. Office of Naval Research Grant N00014-17-1-3021. P. B. S. and T. T. J. acknowledge support from the U.S Office of Naval Research through Grant N00014-16-1-2856. NDBC buoy data are available at the website (http://www.ndbc.noaa.gov); the Spotter wave buoy data and the absolute values of the RMSE analysis are made available in a repository (DOI:10.5281/zenodo.3568426).

\section{References}

Babanin, A. V. (2010). Wind input, nonlinear interactions and wave breaking at the spectrum tail of wind-generated waves, transition from f-4 to f-5 behaviour. Ecological Safety of Coastal and Shelf Zones and Comprehensive Use of Shelf Resources, 21, $173-187$.

Babanin, A. V., McConochie, J., \& Chalikov, D. (2018). Winds near the surface of waves: Observations and modeling. Journal of Physical Oceanography, 48(5), 1079-1088.

Babanin, A. V., \& Soloviev, Y. P. (1998). Variability of directional spectra of wind-generated waves, studied by means of wave staff arrays. Marine and Freshwater Research, 49(2), 89-101. https://doi.org/10.1071/MF96126

Bidlot, J.-R., Holmes, D. J., Wittmann, P. A., Lalbeharry, R., \& Chen, H. S. (2002). Intercomparison of the performance of operational ocean wave forecasting systems with buoy data. Weather and Forecasting, 17(2), 287-310.

Charnock, H. (1955). Wind stress on a water surface. Quarterly Journal of the Royal Meteorological Society, 81(350), 639-640.

Donelan, M. A., Babanin, A. V., Young, I. R., \& Banner, M. L. (2006). Wave-follower field measurements of the wind-input spectral function. Part II: Parameterization of the wind input. Journal of Physical Oceanography, 36(8), 1672-1689. https://doi.org/10.1175/JPO2933.1

Edson, J. B., Jampana, V., Weller, R. A., Bigorre, S. P., Plueddemann, A. J., Fairall, C. W., et al. (2013). On the exchange of momentum over the open ocean. Journal of Physical Oceanography, 43(8), 1589-1610.

Forristall, G. Z. (1981). Measurements of a saturated range in ocean wave spectra. Journal of Geophysical Research: Oceans, 86(C9), 8075-8084. https://doi.org/10.1029/JC086iC09p08075

García-Nava, H., Ocampo-Torres, F. J., Osuna, P., \& Donelan, M. A. (2009). Wind stress in the presence of swell under moderate to strong wind conditions. Journal of Geophysical Research, 114, C12008. https://doi.org/10.1029/2009JC005389

Hansen, C., Katsaros, K. B., Kitaigorodskii, S. A., \& Larsen, S. E. (1990). The dissipation range of wind-wave spectra observed on a lake. Journal of Physical Oceanography, 20(9), 1264-1277.

Holthuijsen, L. H. (2010). Waves in oceanic and coastal waters. Cambridge, U.K.: Cambridge university Press.

Hsu, S. A., Meindl, E. A., \& Gilhousen, D. B. (1994). Determining the power-law wind-profile exponent under near-neutral stability conditions at sea. Journal of Applied Meteorology, 33(6), 757-765.

Johnson, H. K., Højstrup, J., Vested, H. J., \& Larsen, S. E. (1998). On the dependence of sea surface roughness on wind waves. Journal of Physical Oceanography, 28(9), 1702-1716.

Juszko, B.-A., Marsden, R. F., \& Waddell, S. R. (1995). Wind stress from wave slopes using Phillips equilibrium theory. Journal of Physical Oceanography, 25(2), 185-203.

Kitaigorodskii, S. (1983). On the theory of the equilibrium range in the spectrum of wind-generated gravity waves. Journal of Physical Oceanography, 13(5), 816-827.

Kudryavtsev, V. N., \& Makin, V. K. (2001). The impact of air-flow separation on the drag of the sea surface. Boundary-Layer Meteorology, 98(1), 155-171. 
Kuik, A. J., Van Vledder, G. P., \& Holthuijsen, L. H. (1988). A method for the routine analysis of pitch-and-roll buoy wave data. Journal of Physical Oceanography, 18(7), 1020-1034.

Lenain, L., \& Melville, W. K. (2017). Measurements of the directional spectrum across the equilibrium saturation ranges of wind-generated surface waves. Journal of Physical Oceanography, 47(8), 2123-2138.

Long, C. E., \& Resio, D. T. (2007). Wind wave spectral observations in Currituck Sound, North Carolina. Journal of Geophysical Research, 112, C05001. https://doi.org/10.1029/2006JC003835

Makin, V. K., Kudryavtsev, V. N., \& Mastenbroek, C. (1995). Drag of the sea surface. Boundary-Layer Meteorology, 73(1-2), 159-182.

Phillips, O. M. (1985). Spectral and statistical properties of the equilibrium range in wind-generated gravity waves. Journal of Fluid Mechanics, 156, 505-531.

Pickett, M. H., Tang, W., Rosenfeld, L. K., \& Wash, C. H. (2003). QuikSCAT satellite comparisons with nearshore buoy wind data off the US west coast. Journal of Atmospheric and Oceanic Technology, 20(12), 1869-1879.

Raghukumar, K., Chang, G., Spada, F., Jones, C., Janssen, T., \& Gans, A. (2019). Performance characteristics of Spotter, a newly developed real-time wave measurement buoy. Journal of Atmospheric and Oceanic Technology, 36(6), 1127-1141.

Thomson, J., D'Asaro, E. A., Cronin, M. F., Rogers, W. E., Harcourt, R. R., \& Shcherbina, A. (2013). Waves and the equilibrium range at Ocean Weather Station P. Journal of Geophysical Research: Oceans, 118, 5951-5962. https://doi.org/10.1002/2013JC008837

Toba, Y. (1973). Local balance in the air-sea boundary processes. III. On the spectrum of wind waves. Journal of the Oceanographic Society of Japan, 29, 209-220.

Toba, Y., \& Ebuchi, N. (1991). Sea-surface roughness length fluctuating in concert with wind and waves. Journal of the Oceanographic Society of Japan, 47(3), 63-79.

Toba, Y., Okada, K., \& Jones, I. S. F. (1988). The response of wind-wave spectra to changing winds. Part I: Increasing winds. Journal of Physical Oceanography, 18(9), 1231-1240.

Tsagareli, K. N., Babanin, A. V., Walker, D. J., \& Young, I. R. (2010). Numerical investigation of spectral evolution of wind waves. Part I: Wind-input source function. Journal of Physical Oceanography, 40(4), 656-666. https://doi.org/10.1175/2009JPO4370.1

Vincent, C., Thomson, J., Graber, H., \& Collins, C. III. (2019). Impact of swell on the wind-sea and resulting modulation of stress. Progress in Oceanography, 178, 102164.

Voermans, J. J., Rapizo, H., Ma, H., Qiao, F., \& Babanin, A. V. (2019). Air-sea momentum fluxes during Tropical Cyclone Olwyn. Journal of Physical Oceanography, 49, 1369-1379. https://doi.org/10.1175/JPO-D-18-0261.1

Wu, J. (1969). Wind stress and surface roughness at air-sea interface. Journal of Geophysical Research, 74(2), 444-455. https://doi.org/10. 1029/JB074i002p00444

Young, I. R., Verhagen, L. A., \& Banner, M. L. (1995). A note on the bimodal directional spreading of fetch-limited wind waves. Journal of Geophysical Research, 100(C1), 773-778. https://doi.org/10.1029/94JC02218

Zieger, S., Vinoth, J., \& Young, I. R. (2009). Joint calibration of multiplatform altimeter measurements of wind speed and wave height over the past 20 years. Journal of Atmospheric and Oceanic Technology, 26(12), 2549-2564. 


\section{University Library}

\section{- M M I N E R VA A gateway to Melbourne's research publications}

Minerva Access is the Institutional Repository of The University of Melbourne

Author/s:

Voermans, JJ;Smit, PB;Janssen, TT;Babanin, AV

Title:

Estimating Wind Speed and Direction Using Wave Spectra

Date:

2020-02-01

Citation:

Voermans, J. J., Smit, P. B., Janssen, T. T. \& Babanin, A. V. (2020). Estimating Wind Speed and Direction Using Wave Spectra. Journal of Geophysical Research: Oceans, 125 (2), https://doi.org/10.1029/2019JC015717.

Persistent Link:

http://hdl.handle.net/11343/264590 